\title{
Equipo Médico para Clínicas de Mama una Mirada Desde la Práctica de la Ingeniería Clínica
}

\section{Medical Equipment for Breast Clinics a View of Clinical Engineering from Practice}

\section{G. A. Martínez-Chávez}

Instituto Mexicano del Seguro Social

\section{RESUMEN}

El presente trabajo describe la experiencia que involucró la gestión tecnológica de equipo de médico para el establecimiento de la primera y tercera clínica de mama en la delegación CDMX, Sur, del Instituto Mexicano del Seguro Social (IMSS) como una estrategia para el desarrollo de un programa nacional de detección temprana, diagnóstico oportuno y tratamiento con calidad óptima, trato cordial y respeto a los derechos de las pacientes. En este sentido, se exponen los principales argumentos referentes a los lineamientos utilizados para la evaluación de la tecnología medica en el desempeño de los mastógrafos digitales por ser este el equipos más representativo del proyecto, para lo cual se elaboro una cedula de descripción que fue aplicada en seis equipos de mastografía en la cual se vierten los conceptos que intervienen en el análisis de especificaciones técnicas, destacando la respuesta de los detectores, dosimetría, contraste y calidad de la imagen entre los principales aspectos. Teniendo como premisa el compartir las experiencias obtenidas en la creación de dichas clínicas, y el rol del ingeniero biomédico como parte del equipo multidisciplinario, considerado como un gestor del conocimiento respecto a la optimización de los equipos de imagenologia en cada una de las unidades médicas. 


\section{ABSTRACT}

The present work describes the experience which involved the medical management of medical equipment for the establishment of the first and third breast cancer clinic in the Mexican Social Security Institute (IMSS) Delegation CDMX South to development a national program for detection, diagnosis and treatment of breast cancer with optimal quality, friendliness and respect for patients' rights. In this sense it expose the main arguments referring to the guidelines used to validate the performance of digital masthographs, are exposed for being this equipment for the most representative in the project, for which a technical information templates was developed, that applied in six mammography's equipment which are the concepts that intervene in technical specifications analysis, emphasizing the detectors response, dosimetry, contrast and image quality among the main aspects. With the premise of sharing the experiences obtained in the creation of these clinics, and the roll of the biomedical engineer as part multidisciplinary team, and considered optimization of knowledge manager as regarding imaging equipment in each of the medical units.

KEYwORDS: Clinical Engineer; Mammography; Breast Cancer

\section{Correspondencia}

DESTINATARIO: Gustavo Adolfo Martínez Chávez INSTITUCIÓN: Instituto Mexicano del Seguro Social DIRECCIÓN: Av. Insurgentes Sur \#1960, Col. Florida, Del. Álvaro Obregón, C. P. 01030, Ciudad de México, México CORREO ELECTRÓNICO: gustavo.martinezc@imss.gob.mx

\section{Fecha de recepción:}

15 de junio de 2019

Fecha de aceptación:

15 de agosto de 2019 


\section{INTRODUCCIÓN}

La Organización Mundial de la Salud (OMS) estima que año con año en el mundo 1.38 millones de personas son diagnosticadas con cáncer de mama y medio millón son diezmadas por esa causa ${ }^{[1]}$.

La Organización Panamericana de la Salud (OPS) ratifica esta tendencia, siendo la segunda causa de muerte por tumores malignos en los países de America Latina y del Caribe ${ }^{[1]}$.

La creación de las Clínica de Mama "Condesa" y “Tláhuac" en el año 2016 y 2018 por parte del IMSS al Sur de la Ciudad de México, tiene como fundamento la implantación de un modelo de unidad médica especializada que simplifique la detección y diagnóstico del Cáncer de Mama en un periodo de ocho días; situación que se ha desarrollado mediante la integración y colaboración de equipos de trabajo multidisciplinarios que comprende el análisis de diversos aspectos; desde el ámbito social, hasta estudios de genética, estableciendo parámetros útiles para la prevención y diagnostico temprano del cáncer, lo que implica el uso de equipos de ecocardiografía, mamografía y citología y de esta forma poder afinar el pronostico a través de parámetros morfométricos, hecho que facilita la creación de una base de datos para efectuar los análisis a profundidad.

Actualmente, en la bibliografía es posible encontrar diversos procedimientos y metodologías para evaluar y adquirir equipo médico. Sin embargo, en opinión del suscrito, éstas son muy específicas, ya que cuentan con un fundamento importante en las guías de evaluación económica aplicadas en la administración de proyectos de los sistemas de salud, y no concebidas como un aspecto propiamente operativo dentro del ámbito de las competencias de la Ingeniería Biomédica.

Puesto que en ocasiones nos pasa desapercibido que uno de los objetivo principales que se buscan en las actividades de gestión de equipo médico, es la de garantizar la operación de los mismos de forma segura, buscando el máximo rendimiento en su uso, orientada a la disminución de riesgo durante la práctica médica, situación que facilita el seguimiento y supervisión en la operación de los mismos, y que queda implícito dentro de las propias actividades del ingeniero clínico en las unidades.

Por esta razón, se consideró fundamental el analizar las diferentes opciones tecnológicas de equipamiento para el proyecto en cuestión, concluyendo así que la gestión de la tecnología de equipo médico cada día juega un papel más importante para las instituciones prestadoras de servicios de salud, ya que se hace indispensable contar con un correcto proceso de validación, teniendo en cuenta el elevado costo de estos equipos y la implicación que tiene al ser el puente directo entre el personal médico y la población derechohabiente, ya que finalmente el equipo médico es el mecanismo con el cual se diagnostican y emiten resultados clínicos.

\section{METODOLOGÍA}

Ante la diversidad de normas y estándares que rigen y orientan la evaluación de equipos médicos para la detección del cáncer mamario, se hizo necesario buscar consensos, unificar criterios y aplicar una metodología que permitiera la implementación y operación de un sistema práctico de valoración, siendo esencial planear y gestionar la tecnología utilizada en la detección y diagnostico del cáncer de mama, ya que esto implica desde su inicio el administrar su eficiencia, seguridad, costos de mantenimiento y empleo de los equipos de mastografía, al igual que su integración en los sistemas médicos de información con que cuenta el instituto.

La implementación de la metodología tiene su fundamentación en el uso de cedulas descriptivas para el establecimiento de una adecuada planificación en los procesos de adquisición y ministración del equipo médico, en ellas se establecen y se precisan los requerimientos mínimos con que deberán contar cada uno 
de los equipos, cuya descripción es parte fundamental al momento de la planeación del proyecto, considerando que la misma tiene como objetivo guiar al equipo de trabajo con simples tareas como la planificación del proyecto, la definición clara y concisa de objetivos y alcance de la implementación, seguimiento y planes de mitigación de riesgos, revisión de los procesos, configuración, parametrización, pruebas, capacitación, etc.. Una breve descripción de las actividades más relevantes se presenta a continuación:

1. La planeación basada en datos de entrada precisos; resultados comprobados, prioridades claras, análisis completos y decisiones pensadas, considerando para ello los siguientes aspectos.
a. Referencias sobre el tiempo de vida del equipo
b. Mantenimiento
c. Función y criticidad del dispositivo
d. Seguridad
e. Estandarización
f. Estándares de atención
g. Estándares de dispositivos médicos
h. Requisitos legales
i. Estatus tecnológico
j. Compatibilidad de interfaces y redes

2. Acciones referentes al ciclo de vida de los equipos; enfocadas en la ubicación efectiva, seguridad del paciente, educación, capacitación, mantenimiento apropiado, cumplimento de estándares y requisitos legales.

3. Contar con la flexibilidad que permita adicionar factores diferentes a los relativos a los equipos; tales como necesidades de los usuarios, tendencias tecnológicas y reclutamiento médico, entre otros.

4. Finalmente, que sea sencillo de entender y de poner en práctica.
La valoración de cada uno de los criterios antes mencionados aplicados a los equipos de mastografía digital por ser este los más representativos del proyecto, se integraron en una cedula de verificación que comprende lo aspectos funcionales como son la generación de rayos $\mathrm{X}$, colimación, control automático de exposición (CAE), compresión, rejilla y receptor de imagen, dosis y transmisión de la imagen de la unidad de mamografía; destacando el conceso de especificaciones técnicas, al igual que las experiencias vertidas del personal operario, y recomendaciones emitidas por las autoridades normativas del sector salud en México en lo referente al tema de la seguridad radiológica.

La información fue plasmada y analizada en base a los criterios estadísticos de los parámetros de los equipos de mastografía, con la intensión de encontrar coincidencias, en cuanto a la importancia y trascendencia de las características técnicas de los mismos.

Generando datos cuantitativos y cualitativos sobre las variables consideradas como importantes para el funcionamiento en la operación del equipo de mastografía, y que estos fueran acordes a los lineamientos establecido en el proyecto, mismos que se engloban en las guías de practica clínica en lo referente a la respuesta de los detectores, dosimetría, contraste y calidad de la imagen entre los principales aspectos.

\section{RESULTADOS}

Se efectuó la verificación de las características funcionales de seis equipos de mastografía digital, para ello se consideraron diversos aspectos relacionados con visualización de imágenes, documentación para la carga de pacientes, entrenamiento y experiencia del personal, programas de garantía de calidad y cumplimiento con normas internacionales de radio protección; a partir de manuales, catálogos, folletos, instructivos y/o fotografías fue posible el corroborar las especificaciones, y características que de dichos equipos se pueden obtener. 
Para el análisis de la información se destacaron los aspectos relacionados con la seguridad radiológica, el posicionamiento de la glándula mamaria en el mastógrafo, la compresión ejercida en la glándula, la uniformidad en el grosor de la mama, la calidad de la imagen (contraste y nitidez), la ausencia de artefactos, la colimación del haz de radiación y la forma de trasmisión de la imagen en general.

Los resultados obtenidos en la valoración del equipamiento nos indicaron que los sistemas cumplen en su totalidad, obteniéndose como factor a tomar en cuenta que la dosis glandular promedio de los seis equipos se situó en $1 \mathrm{mGy}$, mientras que la norma lo sitúa entre 0.71-1.15 mGy, lo que nos permitió medir el progreso obtenido como un indicador de la tecnología, referente al cumplimiento de la NOM-229-SSA1-2002 en que se plasman los requisitos técnicos y especificaciones técnicas de los equipos en establecimientos de diagnóstico médico con rayos $\mathrm{X}$.

Se observo que los mastógrafos digitales como mecanismos de generación de rayos $\mathrm{X}$ hacen uso de la combinación filtro-ánodo principalmente de molibdeno para mamas pequeñas (compuestas de tejido más adiposo) aproximadamente menores a $5 \mathrm{~cm}$ de espesor reciben una dosis mas reducida.

Las mamas muy grandes (o aquellas de tejidos muy densos) son difíciles de penetrar, con llevan a hacer uso de rayos $\mathrm{X}$ de mayor energía y grandes exposiciones para obtener imágenes aceptables. Motivo por la cual la mayoría de los fabricantes también ofrecen un filtro de rodio para mamas densas, por requerir una mayor energía de penetración.

El tamaño del foco se ubico entre 0.01 y $0.03 \mathrm{~mm}$. para las magnificaciones de las micro calcificaciones, valores que se usan y se establecen en las técnicas de mamografía para reducir la penumbra (borrosidad producida al colocar la mama más lejos del receptor de imagen).
Los metales utilizados como elemento refractario fueron tungsteno, molibdeno y una aleación pesada de tungsteno para ofrecer colimadores de alta precisión con láminas planas, provistas de un rango de grosores de entre 20 y $200 \mu \mathrm{m}$ para los detectores de rayos $\mathrm{X}$, los cuales absorben la radiación dispersa, ayudando así a conseguir imágenes con una calidad alta, ya que con ello se controla el tamaño y forma del haz de radiación, limitando la exposición al tejido mamario comprimido, y permitiendo que el campo de rayos $\mathrm{X}$ pueda extenderse posteriormente, más allá de la pared torácica para incluir el tejido mamario glandular posterior.

Los equipos considerados hacen uso del tubo de rayos $\mathrm{X}$ y del receptor de imagen ubicado en extremos opuestos, lo que permite realizar mamografías en cualquier proyección. La distancia entre la fuente y el receptor de imagen por norma se sitúa en $55 \mathrm{~cm}$. La totalidad de los mastógrafos ofrecen una distancia entre 65 y $70 \mathrm{~cm}$.

Todos los equipos de mastografía incluyeron un control automático de exposición (CAE), el cual tiene la función principal de medir la intensidad y calidad de los rayos $\mathrm{X}$ en el receptor de imagen, lo cual se consigue mediante el uso de arreglos o matrices de CCD (Charged Couple Device, los cuales fueron de silicio o selenio amorfo, absorbiendo directamente los fotones de RX y convirtiéndolos a carga eléctrica. Cada fotodiodo representa igualmente un pixel, ya que la carga total es leída por un contador electrónico de bajo ruido, obteniendo tramas de datos digitales que se envían a un procesador con una respuesta lineal sobre un amplio rango de intensidad de radiación, permitiendo correcciones de los datos y asegurando de esta forma una respuesta uniforme del detector en todo el campo de exploración.

Tal situación propicia las condiciones para que el radiólogo manipule la orientación, la amplificación y el brillo y el contraste de la imagen deseada, facilitando la detección de lesiones del tejido mamario por computadora CAD (Computer Aided Detection), y la transmi- 
TABLA 1. Relación de fabricantes de mastógrafos ampliamente reconocidos como tecnologías rigurosamente digitales, mismos que vienen integrados al equipo de rayos $\mathrm{X}$ y del propio detector

\begin{tabular}{|c|c|c|c|c|c|c|}
\hline \multicolumn{9}{|c|}{ Flat Panel Detectors } \\
\hline Fabricante & Modelo & $\begin{array}{c}\text { Pixel } \\
(\mu \mathrm{m})\end{array}$ & $\begin{array}{c}\text { Dimensiones } \\
(\mathbf{c m})\end{array}$ & $\begin{array}{c}\text { Matriz } \\
(\text { pixels) }\end{array}$ & Bits & Tecnología \\
\hline GE & Senographe DS & 100 & $19 \times 31$ & $1914 \times 2294$ & 14 & CsI-SI \\
\hline Lorad/Hologic & Selenia & 70 & $24 \times 29$ & $3228 \times 4096$ & 14 & $\alpha$-Se \\
\hline Siemens & Mammomat & 70 & $24 \times 29$ & $3328 \times 4084$ & 14 & $\alpha$-Se \\
\hline Planmed & Nuance & 85 & $24 \times 30$ & $2816 \times 3584$ & 13 & $\alpha$-Se \\
\hline IMS & Giotto & 85 & $24 \times 30$ & $2816 \times 3584$ & 13 & $\alpha$-Se \\
\hline Fujifilm & Amulet & 50 & $24 \times 30$ & $4728 \times 5928$ & 13 & $\alpha$-Se \\
\hline
\end{tabular}

sión de imágenes en red, como un medio para separar las funciones de adquisición, almacenamiento y despliegue de las imágenes.

En la Tabla 1 se muestra la relación de fabricantes y las características de los detectores utilizados, todos ellos sistemas que están reconocidos de una manera amplia como tecnologías rigurosamente digitales, mismas que vienen integradas al equipo de rayos $\mathrm{X} \mathrm{y}$ del propio detector.

Todos ellos presentan ventajas notables, entre las que cabe citar: absorción eficiente de la radiación incidente, respuesta lineal para un amplio rango de exposiciones, ruido intrínseco bajo, resolución espacial adecuada, formatos que son apropiados para obtener imágenes de los distintos tamaños de mamas encontrados en la práctica clínica, y sensibilidad adecuada que evite la irradiación excesiva del paciente, con tiempos de exposición aceptables.

Finalmente, este tipo de exámenes mamográficos constituyen una de las exploraciones radiológicas más exigentes, debido a que, simultáneamente, requieren de una alta resolución espacial, un alto contraste y un amplio rango dinámico de los equipos de mastografía que son empleados en los programas de tamizaje mamográfico sobre una población objetivo para detectar una enfermedad en individuos sin signos o síntomas de esa enfermedad.

\section{DISCUSIÓN}

De la experiencia obtenida podemos decir que los resultados del trabajo han tenido una reacción positiva por parte del personal derechohabiente, motivo por el cual se pone a consideración las siguientes situaciones.

a. Los equipos producen una imagen inmediata, sin procesos intermedios de revelado, de lectura ni de ningún otro tipo. Hacen desaparecer los "chasis” y, con ello, permiten construir un entorno puramente digital, limpio, con tiempos muertos menores y con capacidad para incrementar el rendimiento de salas y equipos.

b. Se reducen las dosis a los pacientes o al menos no las incrementan, dado la eficiencia de los detectores empleados en los equipos.

c. Se producen imágenes de calidad muy alta, mucho más estables y con posibilidades muy grandes de adaptación a cada necesidad concreta del paciente. En particular, su resolución de contraste es muy superior a la de los sistemas convencionales. 
d. Es posible optimizar la cadena completa en la obtención de imágenes incluyendo los factores de exposición (kVp, combinación ánodo-filtro, etc.) que se seleccionan en función de las características de la mama (atenuación y espesor). Una consecuencia de ello ha sido el ahorro importante en las dosis en pacientes con mamas gruesas al seleccionar de forma automática espectros de mayor energía.

\section{CONCLUSIONES}

a. Se ha podido reducir los tiempos de respuesta, aunado al aporte social que representa la concepción de la medicina preventiva en la detección y diagnostico oportuno del cáncer de mama.

b. Actualmente estas clínicas esta experimentado una considerable mejora en cuanto al rendimiento de los recursos disponibles, logrando un mayor aprovechamiento de los equipos, un ahorro en costos de mantenimiento, así como la reducción del tiempo en la interrupción del servicio de los equipos de mastografía, lo que se traduce en áreas de oportunidad en la forma con que se hacen los hallazgos de la presencia del cáncer de mama.

c. Finalmente, el presente trabajo pretende hacer referencia para que otras instituciones o centros de salud, fortalezcan sus estrategias que les faciliten mejorar los servicios de atención medica, en que el papel del ingeniero biomédico es la de utilizar los conocimientos de ingeniería para aplicarlos ingeniosamente en todo lo relacionado con el cuidado del paciente, lo cual abarca una amplia gama de actividades y responsabilidades relacionadas con la tecnología medica como un ente neural de todo sistema de salud. 


\section{REFERENCIAS}

[1] Instituto Nacional de Estadística y Geografía, "Estadística a Propósito del Día Mundial de la Lucha Contra el Cáncer de Mama”, del 16 de Octubre de 2015. "consultado 06 marzo 2019 en el siguiente linK: $h t t p: / / w w w . i n e g i . o r g . m x /$ saladeprensa/ aproposito/2015/mamao.pdf

[2] Duffy SW, Tabar L, Chen THH, Smith RA, Holmberg L, Jonsson H, et al. Reduction in Breast Cancer Mortality from Organized Service Screening with Mammography:1. Further Confirmation with Extended Data. Cancer Epidemiol Biomarkers Prev 2006; 15:45-51.

[3] Mandelblatt JS, Cronin KA, Bailey S, Berry DA, de Koning HJ, Draisma $\mathrm{G}$ et al. Effects of Mammography Screening Under Different Screening Schedules: Model Estimates of Potential Benefits and Harms. Ann Intern Med 2009; 738-47.

[4] International Commission on Radiation Units and Measurements. Mammography - Assessment of Image Quality. ICRU Report 82. Oxford University Press. Journal of the ICRU 2009;9(2).

[5] Lewin JM, D’Orsi CJ, Hendrick RE, Moss LJ, Isaacs PK, Karellas A, et al. Clinical comparison of full-field digital mammography and screen-film mammography for detection of breast cancer. Am J Roentgenol 2002;179: 671-7.
[6] Berns EA, Hendrick RE, Cutter GR. Performance comparison of full-field digital mammography to screen-film mammography in clinical practice. Med Phys 2002; 29:830-4.

[7] Lewin JM, Hendrick RE, D’Orsi CJ, Isaacs PK, Moss LJ,et al. Comparison of full-field digital mammography with screen-film mammography for cancer detection: results of 4,945 paired examinations. Radiology2001;218:873-80.

[8] Pisano ED, Gatsonis C, Hendrick E, Yaffe M, Baum JK, Acharyya S, et al. Diagnostic Performance of Digital versus Film Mammography for Breast-Cancer Screening, N Engl J Med 2005;353:1773-83.

[9] Fischer U, Hermann KP, Baum F. Digital mammography: current state and future aspects. Eur Radiol 2006; 16:38-44.

[10] Huda W, Sajewicz AM, Ogden KM, Dance DR. Experimental investigation of the dose and image quality characteristics of a digital mammography imaging system. Med Phys 2003; 30:442-8.

[11] Bern's EA, Hendrick RE, Cutter GR. Optimization of technique factors for a silicon diode array full-field digital mammography system and comparison to screen-film mammography with matched average glandular dose. Med Phys. 2003;30:334-40. 